drug reactions, drug interactions and Clostridium difficile infection. Moreover, there are concerns about inappropriate antibiotic use driving increasing antimicrobial resistance both for individual patients, healthcare institutions and society at large.

NHS England introduced antimicrobial stewardship in 2012 with the aim of optimising antibiotic use for individual patients, preventing the overuse and misuse of antibiotics and minimising the development of resistance at patient, organisation and community levels.

An audit in 2015 showed that 50\% of hospice in-patients received at least one antibiotic course, many of which were used in empirically for a suspected chest or urinary infection during the last two weeks of life. Since this time, we have demonstrated a significant reduction in the use of antimicrobials within our hospice. Initiatives have included; the introduction of 'Start Smart then Focus' guidance, weekly antibiotic dashboards shared with clinical teams, and encouraging earlier discussions with patients and their families/carers. We have also provided education to improve documentation around goals of therapy, increase confidence in stopping antibiotic treatment when considered futile, and improve the use of microbiology specimen requests and results.

We continue to educate and support medical and nursing teams as well as patients and their families to view antibiotic use like other medicines and processes - that significant risks need to be weighed against the potential benefits.

\section{P-141 THE PAIN MANAGEMENT GROUP: AN MDT APPROACH TO PAIN MANAGEMENT IN PALLIATIVE CARE}

Elizabeth Nicholls, Sarah Fielder, Harriet Bush. Countess Mountbatten Hospice, Southampton, UK

\subsection{6/bmjspcare-2019-HUKNC.163}

Background During multi-disciplinary (MDT) patients' meetings, pain management was regularly discussed. Pain was often described as complex and focussed on the pharmaceutical approach, however, it was often noticed that this was not improving a patient's quality of life. Literature highlights the importance of a holistic and MDT approach to pain management (Fielding, Sanford \& Davis, 2013; Phillips, Lovell, Luckett, Agar et al., 2015), but there was little evidence of this in practice except for occasional discussions about exercise. An alternative MDT approach was needed to meet patients' needs and improve quality of life.

Aim To improve patients' management of pain and improve quality of life through an educative programme led by the MDT, with additional support to carers by improving knowledge and teaching management strategies.

Method The five-week out-patient group was developed by a palliative care Consultant, Clinical Nurse Specialist and Occupational Therapist. Each week involved reflection, goal setting, education, exercise and relaxation. Education topics included: What is pain? Understanding medication, Emotional and spiritual pain, Sleep management and The Pain Survival Kit. Outcome measures were used (IPOS, Brief Pain inventory and AKPS). A feedback questionnaire was used for feedback at the end of the five weeks.

Results The outcome measures indicated an improvement in pain, physical abilities, enjoyment of life, relationships and reduced anxieties. Feedback questionnaires reported that patients felt empowered in managing pain especially due to improved understanding. Patients did not require as intensive support from other hospice services.

Conclusions The pain management group improved patients' understanding of pain, medications and sleep, whilst also teaching strategies to increase their control over their pain, enabling a fuller life. The group also produced a reduction in the dependency on health care professionals and hospice services. The group provides an additional treatment option for patients with complex symptoms of total pain.

\section{P-142 EVALUATION OF A HOSPICE BASED INTERVENTIONAL PAIN MANAGEMENT SERVICE FOR PALLIATIVE CARE PATIENTS}

${ }^{1}$ Katy Bruce, ${ }^{2}$ Paul Hart, ${ }^{1}$ Sharon Chadwick. ${ }^{1}$ Hospice of St Francis, Berkhamsted, UK; ${ }^{2}$ West Hertfordshire Hospitals Trust, Watford, UK

\subsection{6/bmjspcare-2019-HUKNC.164}

Background Poorly controlled pain is the most feared symptom of patients at the end of life. It is the most common reason for cancer patients to access emergency out of hours care (Adam, Wassell \& Murchie, 2014). Patients may need to travel to tertiary centres to access specialist pain management. Under-treatment of cancer pain (Deandrea, Montanari, Moja \& Apolone, 2008), late referral (Allsop, Ziegler, Mulvey, Russell et al., 2018) and poor access to pain services have highlighted the need for better integration of Palliative Medicine and Pain Management Services. National guidance for provision of such services for cancer and life-limiting illness has been published (Framework for provision of pain services for adults across the UK with cancer or life-limiting disease, published by Faculty of Pain Medicine, Royal College of Anaesthetists). A hospice based interventional pain management service delivered by a consultant anaesthetist has been set up to address this.

Aim The aim of this evaluation is to:

- establish the characteristics of patients accessing the service;

- describe interventions delivered;

- perform a qualitative assessment of the impact of interventions;

- identify how access to the service can be improved;

- assess and report the value of providing a local, hospicebased pain intervention service.

Methods A retrospective case note review will be conducted and the following data collected from the narrative; number/demographics of patients, source of referral, interventions provided, patient perceived benefit, prescribed analgesics at referral as a surrogate marker of pain intractability.

Results It is anticipated that most patients are seen at a late stage in their illness and that the pool of referrers will be small. It is likely that patients referred will already be taking several analgesics due to intractable pain.

Conclusions Data will be used to assess the impact of the service, obstacles to setting up and delivering the service and the overall value of providing a local hospice-based interventional pain management service. A likely outcome will be targeting of referrers to facilitate earlier access to the service and to establish better communication between potential referrers and the service. 